\title{
Elevated p53 expression levels correlate with tumor progression and poor prognosis in patients exhibiting esophageal squamous cell carcinoma
}

\author{
KATE HUANG $^{1 *}$, LIN CHEN ${ }^{2 *}$, JILIANG ZHANG $^{2}$, ZHI WU $^{2}$, LINHUA LAN $^{2}$, \\ $\mathrm{LU}_{\mathrm{WANG}}{ }^{2}, \mathrm{BIN} \mathrm{LU}^{2}$ and YONGZHANG LIU ${ }^{2}$ \\ ${ }^{1}$ Department of Pathology, The First Affiliated Hospital of Wenzhou Medical University; \\ ${ }^{2}$ Department of Biochemistry and Molecular Biology, Attardi Institute of Mitochondrial Biomedicine, \\ Zhejiang Provincial Key Laboratory of Medical Genetics, School of Laboratory Medicine and Life Sciences, \\ Wenzhou Medical University, Wenzhou, Zhejiang 325035, P.R. China
}

Received January 26, 2014; Accepted June 19, 2014

DOI: $10.3892 / 01.2014 .2343$

\begin{abstract}
Esophageal squamous cell carcinoma (ESCC) is the most common histological subtype of esophageal cancer and one of the most aggressive types of malignancy, with a high rate of mortality. Early diagnosis and treatment may improve the prognosis of ESCC and, thus, survival rates. As a significant tumor suppressor, p53 is closely associated with apoptosis and the differentiation of cancer cells. The present study evaluated the expression levels of the p53 protein and the clinical significance in patients presenting with ESCC. The $\mathrm{p} 53$ protein expression level of 64 paired ESCC and tumor-adjacent normal tissues was evaluated using western blot analysis. In addition, immunohistochemistry (IHC) was performed to detect the p53 expression level in specimens from 118 paraffin-embedded cancerous tissues. The correlation of the p53 expression level with the clinicopathological parameters and prognosis of the ESCC patients was also analyzed. The p53 protein was identified to be highly expressed in the ESCC tissue, with western blot analysis demonstrating that the expression level of p53 in the cancerous tissue was 1.89 times that of the tumor-adjacent normal tissue $(\mathrm{P}<0.001)$; furthermore, IHC indicated that there
\end{abstract}

Correspondence to: Professor Yongzhang Liu or Professor Bin Lu, Department of Biochemistry and Molecular Biology, Attardi Institute of Mitochondrial Biomedicine, Zhejiang Provincial Key Laboratory of Medical Genetics, School of Laboratory Medicine and Life Sciences, Wenzhou Medical University, 1 North Central Avenue, 4B-406 Tong De Building, Wenzhou, Zhejiang 325035, P.R. China

E-mail: lyz@wzmc.edu.cn

E-mail: lubmito@wzmc.edu.cn

*Contributed equally

Key words: esophageal squamous cell carcinoma, p53, immunohistochemistry, western blot, prognosis was a marked positive expression of p53 in the ESCC tissue $(49.15 \%)$. The expression level of p53 protein was identified to be significantly correlated with the tumor grade $(\mathrm{P}<0.001)$, $\mathrm{N}$ stage $(\mathrm{P}=0.010)$. Additionally, the higher level of $\mathrm{p} 53$ expression was found to be associated with a poor survival rate in the ESCC patients $(\mathrm{P}=0.0404)$. The univariate analysis showed that the survival time of patients was significantly correlated with the $\mathrm{T}$ stage $(\mathrm{RR}=3.886, \mathrm{P}<0.001)$, $\mathrm{N}$ stage (lymph node metastasis; $\mathrm{RR}=3.620, \mathrm{P}<0.001)$ and $\mathrm{TNM}$ stage $(\mathrm{RR}=3.576$, $\mathrm{P}<0.001)$. Furthermore, the multivariate analysis revealed that the $\mathrm{T}$ stage $(\mathrm{RR}=3.988, \mathrm{P}<0.001)$ and $\mathrm{N}$ stage $(\mathrm{R}=4.240$, $\mathrm{P}=0.004)$ significantly influenced the overall survival of the ESCC patients.

\section{Introduction}

Human esophageal squamous cell carcinoma (ESCC) is one of the most aggressive types of cancer and is ranked as the sixth most frequent cause of cancer-associated mortality in the world, with a high incidence in northern China, South Africa, Turkey and Iran (1-3). Furthermore, ESCC constitutes $7 \%$ of all gastrointestinal cancers and is the predominant histological subtype of esophageal cancer, comprising $\sim 70 \%$ of cases worldwide. Recently, progress in early diagnosis, surgery, and chemo- and radiotherapy has led to an increase in the eight-year overall survival rate of ESCC patients, however, improving the prognosis of ESCC patients remains a challenge.

Tumor suppressor p53, encoded by the $p 53$ gene located at chromosome 17q13.1, is highly associated with a poor prognosis in human cancers $(4,5)$. It is well known that the p53 protein may induce cell apoptosis and regulate cell proliferation. Mutation of the $p 53$ gene results in the loss of its ability to induce cell death, which leads to uncontrolled cell growth, thus, promoting tumorigenesis $(6,7)$.

In the present study, the overexpression of p53 in the nucleus of the ESCC patient tissues was examined via tissue microarray (TMA), which incorporated 118 ESCC specimens, as well as using western blotting to analyze 64 samples of freshly frozen tissues from ESCC patients. 
The correlation between the p53 protein expression level, and tumor progression and prognosis of ESCC patient was evaluated, which may provide further data for predicting the progression and prognosis in patients with ESCC.

\section{Patients and methods}

Patients and tissue samples. A total of 64 paired tissue samples, including tumor tissue and the adjacent non-cancerous tissue, were collected from ESCC patients who underwent surgery at the Department of Cardiothoracic Surgery, the First Affiliated Hospital of Wenzhou Medical University (Wenzhou, China) between May 2012 and September 2013. The tissues were immediately frozen in liquid nitrogen following surgery and stored at $-80^{\circ} \mathrm{C}$ until undergoing western blot analysis to detect p53 expression levels. Written informed consent for experimental use of the specimens was obtained from all patients and the study was approved by the Board and Ethics Committee of Wenzhou Medical University (Wenzhou, China). All the patients were clinically and pathologically confirmed to exhibit ESCC, and the tumor tissues were classified according to the American Joint Committee on Cancer/Union Internationale Contre le Cancer and were histologically graded in accordance with the World Health Organization classification $(8,9)$.

Protein extraction and western blot analysis. The total protein from the 64 paired tissue samples was homogenized using a homogenizer (Polytron PT-MR2100; Kinematica AG, Luzern, Switzerland) in $1.5 \mathrm{ml}$ tissue radio-immunoprecipitation assay lysis buffer (50 mM Tris [pH 7.4], $150 \mathrm{mM}$ $\mathrm{NaCl}, 1.0 \%$ Triton $\mathrm{X}-100,1 \%$ sodium deoxycholate and $0.1 \%$ SDS; Beyotime Institue of Biotechnology, Shanghai, China) containing protease inhibitor cocktail (Roche Applied Science, Indianapolis, IN, USA), $1 \mathrm{mM} \mathrm{NaF}$ and $1 \mathrm{mM} \mathrm{Na}_{3} \mathrm{VO}_{4}$. Tissue homogenates were incubated on ice for $15 \mathrm{~min}$, centrifuged (Centrifuge 5417R; Eppendorf, Hauppauge, NY, USA) at $18,000 \mathrm{x}$ g for $20 \mathrm{~min}$ at $4^{\circ} \mathrm{C}$ and the supernatants were collected. The protein concentration was subsequently quantified using a BCA Protein assay kit (Thermo Fisher Scientific, Waltham, MA, USA). A total of $20 \mathrm{mg}$ protein from each sample was separated by $10 \%$ SDS-PAGE (Bio-Rad, Hercules, CA, USA) and transferred onto a nitrocellulose membrane (Bio-Rad). Immunoblot analysis was subsequently performed with monoclonal rabbit anti-human p53 (Proteintech Group, Wuhan, China) and monoclonal mouse anti-human actin (Abmart Inc., Shanghai, China) antibodies. The horseradish peroxidase-conjugated secondary antibodies were obtained from Abmart Inc. The signals were visualized using an electrochemiluminescence system (Thermo Fisher Scientific) according to the manufacturer's instructions and the optical density was quantified using the National Institutes of Health ImageJ software (http://imagej.nih.gov/ij/download/).

TMA and immunohistochemistry (IHC). An ESCC TMA, containing a total of 118 formalin-fixed paraffin-embedded tissue samples, was constructed according to a previously described method (10). IHC was also performed according to a previously described method (11). Briefly, the sections were deparaffinized in xylene and rehydrated through a gradient concentration of alcohol. The endogenous peroxidase activity was inactivated, non-specific staining was blocked by $5 \%$ normal goat serum and all sections were incubated with anti-p53 antibody (1:100; Abmart Inc.) overnight at $4^{\circ} \mathrm{C}$. The slides were incubated with biotin-labeled goat anti-rabbit immunoglobulin $\mathrm{G}$ and further incubated with streptavidin peroxidase solution (SABC kit, Boster Biological Technology, Ltd., Wuhan, China). The staining was visualized by reaction with 3, 3'-di-aminobenzidine (Boster Biological Technology, Ltd.) in phosphate-buffered saline [PBS; Dycent Biotech (Shanghai) Co. Ltd., Shanghai, China] with $0.05 \% \mathrm{H}_{2} \mathrm{O}_{2}$ for $5 \mathrm{~min}$ at room temperature. Control staining was performed by staining the same TMA (duplicate) with PBS rather than anti-p53 and no immunostaining was observed. The slides were counter-stained with hematoxylin, washed in double-distilled $\mathrm{H}_{2} \mathrm{O}$ and mounted with resinous mounting medium. The TMA were scored separately by two pathologists who had no prior knowledge of the clinicopathological status of the specimens on the TMA.

Assessment of IHC. Histopathological sections were microscopically examined (Nikon ECLIPSE 80i; Nikon Corporation, Tokyo, Japan) and scored by two independent pathologists, who were blinded to the clinical data pertaining to the patients. The IHC staining of mutant (MT)p53 was assessed according to the immune-reactive score (IRS) as described previously $(12,13)$ with slight adjustments, which evaluated the percentage of positive cells and the staining intensity. The percentage of positive cells was scored as follows: $1, \leq 10 \%$ positive cells; $2,11-49 \% ; 3,50-79 \%$; and $4, \geq 80 \%$ (14). The staining intensity was graded as 0 , negative; 1 , weak; 2 , moderate; and 3 , strong. The two scores were multiplied and the IRS (a value between 0 and 12) was determined as low or high, which corresponded to IRS values of $\leq 6$ and $>6$, respectively.

Statistical analysis. The optical density of the western blot signals was quantified using the National Institutes of Health ImageJ software and all statistical analyses were carried out using the SPSS 13.0 statistical software package (SPSS Inc., Chicago, IL, USA). The expression level of p53 was quantified relative to $\beta$-actin and the differences between the cancer tissues and adjacent normal tissues in p53 protein expression levels were compared using Student's t-test. The $\chi^{2}$ test was performed to evaluate the correlation between the clinicopathological features of the patients and the p53 expression level, which was observed by IHC. Kaplan-Meier survival analysis was used to evaluate the patient prognosis and the eight-year survival rate of the ESCC patients was obtained using the life table method. A univariate analysis was plotted using the Kaplan-Meier method and Cox regression analysis was used to evaluate the correlation between risk of ESCC and clinicopathological parameters, including p53 expression. $\mathrm{P} \leq 0.05$ was considered to indicate a statistically significant difference.

\section{Results}

Increased level of 53 expression was observed in ESCC tissues when compared with paired non-neoplastic tissues. In the present study, the p53 protein expression level of 64 paired tumor tissues and non-neoplastic tissues was analyzed using western blot. The result demonstrated that the p53 expression level in the 
A

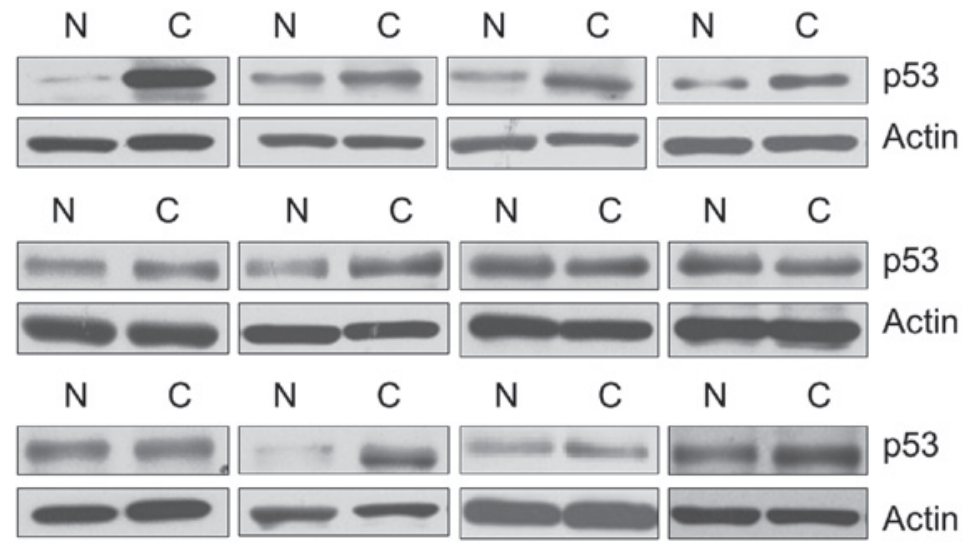

B

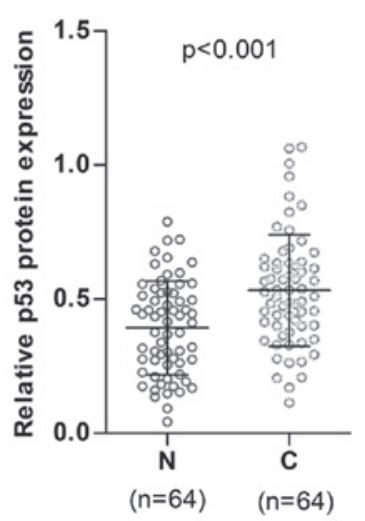

Figure 1. p53 protein expression in esophageal squamous cell carcinoma (ESCC) patient tissues. (A) Western blot analysis of p53 protein expression in ESCC patient tumor tissue (denoted as C) and matched tumor-adjacent non-neoplastic tissue (denoted as N). (B) The quantitative results of western blot analysis demonstrated that p53 expression was increased in the tumor tissue when compared with the matched non-neoplastic tissue of the ESCC patients. ( $\mathrm{n}=64, \mathrm{P}<0.001$ ).

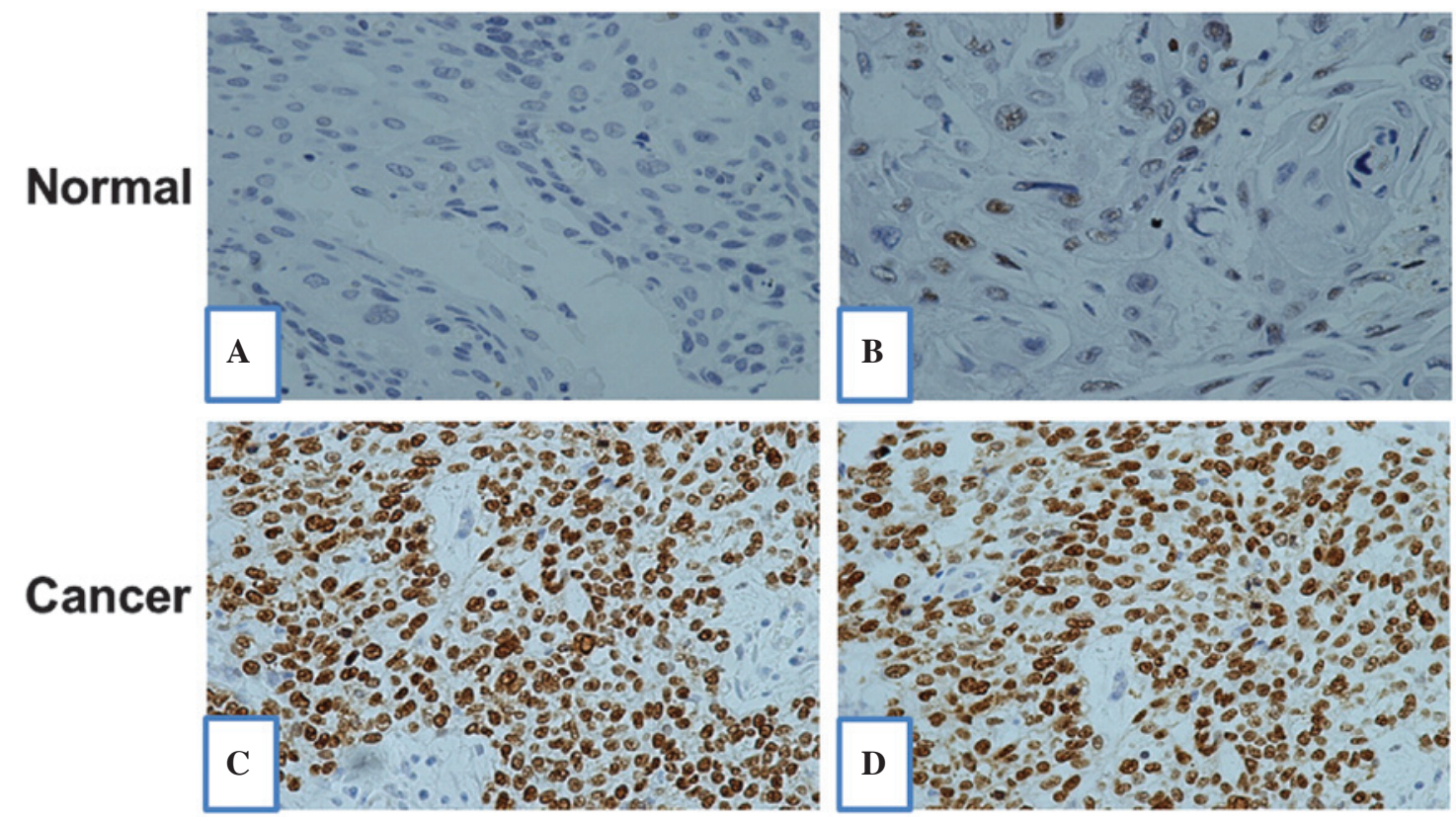

Figure 2. Representative immunohistochemistry for p53 in esophageal squamous cell carcinoma tissues (magnification, x400). (A and B) Tumor-adjacent non-neoplastic tissue and (C and D) tumor tissue. The p53 protein is predominantly localized in the nucleus of the cancer cells.

ESCC tissues was significantly higher than that in the matched non-neoplastic tissues. The $\mathrm{p} 53$ protein in the tumor tissue of the ESCC patients was found to be 1.89 times that of the matched non-neoplastic tissues ( $\mathrm{n}=64, \mathrm{P}<0.001$; Fig. $1 \mathrm{~A}$ and B).

IHC of p53 expression levels in ESCC and matched non-neoplastic tissues. IHC of ESCC TMA was conducted to further evaluate the level of p53 protein expression in the ESCC tissues. IHC revealed that the $\mathrm{p} 53$ protein was predominantly localized in the nucleus (Fig. 2A) and the expression level of p53 in the ESCC tissue was identified to be significantly higher when compared with that in the adjacent normal tissues (Fig. 2A).

Association of the 553 protein expression level with clinicopathological features. A total of 118 ESCC patient tissue samples were used to construct the TMA, including 96 males and 22 females (age range, 26-79 years; median, 63 years). The IHC staining for p53 (MTp53) demonstrated low and high levels of p53 expression in 60 (50.8\%) and 58 (49.15\%) samples, respectively. The level of p53 protein expression was found to correlate with the pathological grade $(\mathrm{P}<0.001)$ and $\mathrm{N}$ stage $(\mathrm{P}=0.007)$, however, not with patient age, gender, history of alcohol consumption and smoking, $\mathrm{T}$ stage or TNM stage (Table I).

Survival analysis. The patients with clear follow-up data were used for the survival analysis. Out of 118 patients, there were 58 cases that exhibited a high expression level of p53 $(49.15 \%)$, and 60 cases $(50.85 \%)$ that exhibited a low expression level of p53. The eight-year survival rate was $36.21 \%$ 
Table I. Association between p53 expression and various clinicopathological factors of esophageal squamous cell carcinoma patients.

\begin{tabular}{|c|c|c|c|c|}
\hline \multirow[b]{2}{*}{ Characteristic } & \multirow{2}{*}{$\begin{array}{c}\text { Total, } \\
\mathrm{n}=118\end{array}$} & \multicolumn{2}{|c|}{ p53 protein expression } & \multirow[b]{2}{*}{ P-value } \\
\hline & & Low, $n=60$ & High, $n=58$ & \\
\hline Gender & & & & 0.391 \\
\hline Male & 96 & 47 & 49 & \\
\hline Female & 22 & 13 & 9 & \\
\hline Age & & & & 0.732 \\
\hline$<60$ & 49 & 24 & 25 & \\
\hline$\geq 60$ & 69 & 36 & 33 & \\
\hline Smoker & & & & 0.721 \\
\hline Yes & 63 & 33 & 30 & \\
\hline No & 55 & 27 & 28 & \\
\hline Alcohol consumer & & & & 0.732 \\
\hline Yes & 69 & 36 & 33 & \\
\hline No & 49 & 24 & 25 & \\
\hline Pathological grade & & & & $<0.001^{\mathrm{b}}$ \\
\hline G1 & 37 & 29 & 8 & \\
\hline $\mathrm{G} 2$ & 56 & 27 & 29 & \\
\hline G3 & 25 & 4 & 21 & \\
\hline T stage ${ }^{\mathrm{a}}$ & & & & 0.062 \\
\hline $\mathrm{T} 1$ & 23 & 17 & 6 & \\
\hline $\mathrm{T} 2$ & 36 & 14 & 22 & \\
\hline $\mathrm{T} 3$ & 56 & 28 & 28 & \\
\hline $\mathrm{T} 4$ & 3 & 1 & 2 & \\
\hline N stage ${ }^{a}$ & & & & $0.010^{\mathrm{b}}$ \\
\hline N0 & 67 & 41 & 26 & \\
\hline $\mathrm{N} \geq 1$ & 51 & 19 & 32 & \\
\hline
\end{tabular}

${ }^{a}$ According to the TNM Classification of Malignant Tumours. ${ }^{b} \mathrm{P}<0.05$ was considered to indicate a statistically significant difference. The $\chi^{2}$ test was used to analyze the association between p53 expression levels and clinicopathological characteristics. T, tumor; N, necrosis; M, metastasis.

Table II. Univariate analysis and multivariate analysis identifies the factors that influence the overall survival rate of esophageal squamous cell carcinoma patients.

\begin{tabular}{|c|c|c|c|c|c|c|}
\hline \multirow[b]{2}{*}{ Variable } & \multicolumn{3}{|c|}{ Univariate analysis } & \multicolumn{3}{|c|}{ Multivariate analysis } \\
\hline & $\mathrm{RR}$ & $95 \% \mathrm{CI}$ & P-value & $\mathrm{RR}$ & $95 \% \mathrm{CI}$ & P-value \\
\hline p53 & 1.646 & $0.987-2.745$ & 0.056 & 1.282 & $0.736-2.233$ & 0.381 \\
\hline Age & 1.007 & $0.979-1.035$ & 0.652 & 1.012 & $0.984-1.040$ & 0.408 \\
\hline Gender & 1.282 & $0.651-2.523$ & 0.472 & 1.160 & $0.501-2.685$ & 0.728 \\
\hline Smoker & 1.095 & $0.661-1.811$ & 0.725 & 1.755 & $0.756-4.077$ & 0.191 \\
\hline Alcohol consumer & 0.948 & $0.570-1.577$ & 0.948 & 0.531 & $0.224-1.259$ & 0.151 \\
\hline Pathological grade & 1.342 & $0.749-2.404$ & 0.323 & 1.057 & $0.571-1.955$ & 0.860 \\
\hline T stage $^{\mathrm{a}}$ & 3.886 & $2.256-6.696$ & $<0.001$ & 3.988 & 1.969-8.077 & $<0.001$ \\
\hline $\mathrm{N}$ stage $^{\mathrm{a}}$ & 3.620 & 2.149-6.099 & $<0.001$ & 4.240 & $1.580-11.378$ & 0.004 \\
\hline TNM stage ${ }^{a}$ & 3.576 & $2.144-5.963$ & $<0.001$ & 0.596 & $0.207-1.716$ & 0.381 \\
\hline
\end{tabular}

${ }^{a}$ According to the TNM Classification of Malignant Tumours. The Cox proportional hazards model was used to identify the factors that had a significant influence on overall survival. $\mathrm{P}<0.05$ was considered to indicate a statistically significant difference. RR, relative risk; CI, confidence interval; T, tumor; $\mathrm{N}$, necrosis; $\mathrm{M}$, metastasis. 


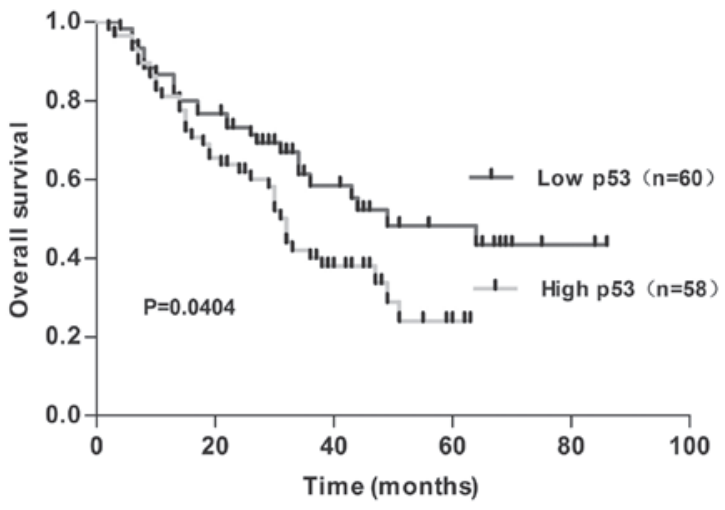

Figure 3. Correlation between $\mathrm{p} 53$ protein expression status and prognosis of esophageal squamous cell carcinoma (ESCC) patients. The Kaplan-Meier overall survival curve of ESCC patients $(n=118)$ according to $p 53$ protein expression level demonstrated that ESCC patients exhibiting high levels of p53 protein expression are associated with a poor overall survival. $\mathrm{P}=0.0404$

(21/58), 56.67\% (34/60), in the high p53 expression group and low p53 expression group, respectively. Survival curves were obtained using the Kaplan-Meier analysis and the log-rank test was used to compare differences in survival between the two groups. According to the survival analysis, it was found that the eight-year survival rate of the group with low levels of p53 protein expression was higher than the group with high levels of p53 protein expression ( $\mathrm{P}=0.0404$; Fig. 3$)$.

Assessment of ESCC risk factors. The univariate analysis and multivariate analysis were used to evaluate the influence of various parameters on the disease-free survival rate of ESCC patients. The ESCC risk factors, including the p53 expression level, patient age, gender, TNM stage, pathological grade, $\mathrm{N}$ stage, $\mathrm{T}$ stage, and history of smoking and alcohol consumption were taken into account (Table II). The univariate analysis demonstrated that the survival time of patients was significantly correlated with the $\mathrm{T}$ stage $(\mathrm{RR}=3.886, \mathrm{P}<0.001)$, $\mathrm{N}$ stage $(\mathrm{R} R=3.620, \mathrm{P}<0.001)$ and $\mathrm{TNM}$ stage $(\mathrm{RR}=3.576$, $\mathrm{P}<0.001)$. Furthermore, multivariate analysis revealed that the $\mathrm{T}$ stage $(\mathrm{RR}=3.988, \mathrm{P}<0.001)$ and $\mathrm{N}$ stage $(\mathrm{R}=4.240$, $\mathrm{P}=0.004)$ were significant and independent prognostic factors for ESCC patients.

\section{Discussion}

The human p53 gene is located at chromosome 17p13.1 and encodes the p53 protein, which is composed of 393 amino acids. The $p 53$ gene is a member of a highly conserved family that contains at least another two genes, $p 63$ and $p 73$. The wild-type (WT)p53 protein contains 393 amino acids and p53 is a tumor suppressor that has a close association with numerous types of human cancer; the mutation or loss of the $p 53$ gene can be identified in $>50 \%$ of all human cancers $(15,16)$. p53 is involved in the regulation of the cell cycle, as well as inducing a variety of activities to maintain the genomic stability, cellular senescence $(17,18)$ and apoptosis (19). Under normal conditions, p53 protein levels are maintained at a very low level unless the cells are activated by signals from DNA damage, as well as certain other cellular stresses (20). The response to DNA damage and cellular stresses is the upregulation of the p53 protein expression level, which leads to cell cycle arrest, DNA repair or apoptosis. Thus, p53 is critical in the inhibition of malignant cancer cell division.

There are two types of p53 proteins, WTp53 and MTp53. WTp53 is a tumor suppressor, which prevents the proliferation of tumor cells; MTp53 causes issues with the regulation of the cell cycle, resulting in uncontrolled cell growth that promotes tumorigenesis. WTp53 has a particularly short half-life and is difficult to detect in normal cells. Conversely, MTp53 is markedly more stable, with a longer half-life, which favors detection by IHC. Previous studies have detected the p53 mutation using IHC and were able to define the tissue via the strong staining of the p53 protein as MTp53 (21-23). Based on this finding, the point mutation in the $p 53$ gene was associated with p53 protein stabilization. The majority of human cancers may be detected via the upregulation of the p53 protein, including liposarcoma (24), colorectal cancer (25), breast carcinomas (26) and endometrial carcinomas (27). Recently, Zhu et al (28) demonstrated that the knock-down of MTp53 using small interfering RNA induced cell cylce arrest and triggered apoptosis in bladder cancer cells.

Chava et al (29) performed IHC in archival tissue samples to evaluate the expression levels of fragile histidine triad (FHIT) and the $\mathrm{p} 53$ protein. The study indicated that the level of $p 53$ gene expression was eight times that which was observed in the normal tissues. The results showed that FHIT and p53 were well correlated with SCC. As the study only involved 23 ESCC samples to perform the IHC, a greater number of samples are required to improve the evaluation of the correlation of the p53 protein with ESCC. In the present study, western blot analysis was performed using tissues, which were snap-frozen in liquid nitrogen and stored at $-80^{\circ} \mathrm{C}$. The results demonstrated that the expression level of p53 protein in the cancer tissues was 1.89 times than that in the normal tissue. In addition, IHC analysis was conducted and the results demonstrated that the tumor tissues of the ESCC patients exhibited strong p53 protein staining, whereas the matched tumor-adjacent tissues exhibited weak p53 staining. Therefore, the upregulation of p53 in ESCC patient tissues has a significant role in esophageal carcinomaproliferation.

ESCC tumorigenesis is a complex process, which is affected by various factors. The pathogenesis of ESCC remains unclear, and numerous studies indicate that ESCC is associated with multi-factor and multi-gene mutations. However, previous studies have shown that environmental and lifestyle factors, such as smoking, alcohol consumption, lack of fruit and vegetable intake, or an excess of pickled foods are potential factors that may lead to esophageal cancer (29-33). In the present study, the Cox proportional hazards model was used during the statistical analysis, and revealed that the patient age, gender, clinical and pathological stages, and the presence or absence of a history of alcohol and tobacco use did not result in significant differences with regard to an association with prognosis; however, the results of univariate analysis showed that the $\mathrm{T}$ stage, $\mathrm{N}$ stage and TNM stage were significantly correlated with the prognosis of patient survival. Additionally, multivariate analysis revealed that the $\mathrm{T}$ and $\mathrm{N}$ stages correlated with ESCC patient survival.

In conclusion, the present results further demonstrated that p53 (MTp53) is overexpressed in the tumor tissue of ESCC patients, which leads to transcriptional regulation dysfunction and uncontrolled cell growth. Therefore, p53 may be used as a 
specific therapeutic target for the treatment of ESCC and as a biomarker for the diagnosis of ESCC.

\section{Acknowledgements}

The authors would like to thank Dr Charles Reichman for the critical review of the manuscript. The current study was partially supported by the National Natural Science Foundation of China (grant nos. 31070710 and 31171345), the Zhejiang Qianjiang Talent Project B Grant (grant no. 2010R10045), which was awarded to Professor Bin Lu, and the Natural Science Foundation of Zhejiang Province (grant no. Y2110097), which was awarded to Professor Yongzhang Liu.

\section{References}

1. Song QK, Li J, Jiang HD, He YM, Zhou XQ and Huang CY: Esophageal cancer mortality during 2004-2009 in Yanting County, China. Asian Pac J Cancer Prev 13: 5003-5006, 2012.

2. Blot WJ: Esophageal cancer trends and risk factors. Semin Oncol 21: 403-410, 1994.

3. Brooks-Brunn JA: Esophageal cancer: an overview. Medsurg Nurs 9: 248-254, 2000.

4. Cho H, Ha SY, Park SH, Park K and Chae YS: Role of p53 gene mutation in tumor aggressiveness of intracranial meningiomas. J Korean Med Sci 14: 199-205, 1999.

5. Murata A, Baba Y, Watanabe M, et al: p53 immunohistochemical expression and patient prognosis in esophageal squamous cell carcinoma. Med Oncol 30: 728, 2013.

6. Cardin R, Piciocchi M, Tieppo C, et al: Oxidative DNA damage in Barrett mucosa: correlation with telomeric dysfunction and p53 mutation. Ann Surg Oncol 20 (Suppl 3): S583-S589, 2013.

7. Di Agostino S, Strano S and Blandino G: Gender, mutant p53 and PML: a growing 'affaire' in tumor suppression and oncogenesis. Cell Cycle 12: 1824-1825, 2013.

8. Guinan P, Sobin LH, Algaba F, et al: TNM staging of renal cell carcinoma: Workgroup No. 3. Union International Contre le Cancer (UICC) and the American Joint Committee on Cancer (AJCC). Cancer 80: 992-993, 1997.

9. Sobin LH and Fleming ID: TNM Classification of Malignant Tumors, fifth edition (1997). Union Internationale Contre le Cancer and the American Joint Committee on Cancer. Cancer 80: 1803-1804, 1997.

10. Nocito A, Bubendorf L, Tinner EM, et al: Microarrays of bladder cancer tissue are highly representative of proliferation index and histological grade. J Pathol 194: 349-357, 2001.

11. Nie X, Li M, Lu B, et al: Down-regulating overexpressed human Lon in cervical cancer suppresses cell proliferation and bioenergetics. PloS One 8: e81084, 2013.

12. Remmele W and Stegner HE: Recommendation for uniform definition of an immunoreactive score (IRS) for immunohistochemical estrogen receptor detection (ER-ICA) in breast cancer tissue. Pathologe 8: 138-140, 1987 (In German).

13. Cheng AN, Jiang SS, Fan CC, et al: Increased Cdc7 expression is a marker of oral squamous cell carcinoma and overexpression of $\mathrm{Cdc} 7$ contributes to the resistance to DNA-damaging agents. Cancer Lett 337: 218-225, 2013.

14. Bolander A, Agnarsdóttir M, Strömberg S, et al: The protein expression of TRP-1 and galectin-1 in cutaneous malignant melanomas. Cancer genomics Proteomics 5: 293-300, 2008.
15. Grelewski PG and Bar JK: The role of $\mathrm{p} 53$ protein and MMP-2 tumor/stromal cells expression on progressive growth of ovarian neoplasms. Cancer Invest 31: 472-479, 2013.

16. Lee JY, Kim HJ, Yoon NA, et al: Tumor suppressor p53 plays a key role in induction of both tristetraprolin and let-7 in human cancer cells. Nucleic Acids Res 41: 5614-5625, 2013.

17. Krell J, Frampton AE, Colombo T, et al: The p53 miRNA interactome and its potential role in the cancer clinic. Epigenomics 5: 417-428, 2013.

18. Gu Z, Jiang J, Tan W, et al: p53/p21 Pathway involved in mediating cellular senescence of bone marrow-derived mesenchymal stem cells from systemic lupus erythematosus patients. Clin Dev Immunol 2013: 134243, 2013.

19. Zhou Y and Ho WS: Combination of liquiritin, isoliquiritin and isoliquirigenin induce apoptotic cell death through upregulating p53 and p21 in the A549 non-small cell lung cancer cells. Oncol Rep 31: 298-304, 2014.

20. Formigari A, Gregianin E and Irato P: The effect of zinc and the role of p53 in copper-induced cellular stress responses. J Appl Toxicol 33: 527-536, 2013.

21. Biramijamal F, Allameh A, Mirbod P, Groene HJ, Koomagi R and Hollstein M: Unusual profile and high prevalence of p53 mutations in esophageal squamous cell carcinomas from northern Iran. Cancer Res 61: 3119-3123, 2001.

22. Guimaraes DP and Hainaut P: TP53: a key gene in human cancer. Biochimie 84: 83-93, 2002.

23. Taghavi N, Biramijamal F, Sotoudeh M, et al: Association of p53/p21 expression with cigarette smoking and prognosis in esophageal squamous cell carcinoma patients. World J Gastroenterol 16: 4958-4967, 2010.

24. Chiarugi V and Ruggiero M: Role of three cancer 'master genes' p53, bcl2 and c-myc on the apoptotic process. Tumori 82: 205-209, 1996

25. Huerta S, Gao X, Dineen S, Kapur P, Saha D and Meyer J: Role of p53, Bax, p21, and DNA-PKcs in radiation sensitivity of HCT-116 cells and xenografts. Surgery 154: 143-151, 2013.

26. Jung SY, Jeong J, Shin SH, et al: Accumulation of p53 determined by immunohistochemistry as a prognostic marker in node negative breast cancer; analysis according to St Gallen consensus and intrinsic subtypes. J Surg Oncol 103: 207-211, 2011.

27. Koshiyama M, Konishi I, Wang DP, et al: Immunohistochemical analysis of p53 protein over-expression in endometrial carcinomas: inverse correlation with sex steroid receptor status. Virchows Arch A Pathol Anat Histopathol 423: 265-271, 1993.

28. Zhu HB, Yang K, Xie YQ, Lin YW, Mao QQ and Xie LP: Silencing of mutant p53 by siRNA induces cell cycle arrest and apoptosis in human bladder cancer cells. World J Surg Oncol 11: $22,2013$.

29. Chava S, Mohan V, Shetty PJ, et al: Immunohistochemical evaluation of p53, FHIT, and IGF2 gene expression in esophageal cancer. Dis Esophagus 25: 81-87, 2012.

30. Launoy G, Milan C, Day NE, Pienkowski MP, Gignoux M and Faivre J: Diet and squamous-cell cancer of the oesophagus: a French multicentre case-control study. Int J Cancer 76: 7-12, 1998.

31. Sammon AM and Alderson D: Diet, reflux and the development of squamous cell carcinoma of the oesophagus in Africa. Br J Surg 85: 891-896, 1998.

32. Morse DE, Pendrys DG, Katz RV, et al: Food group intake and the risk of oral epithelial dysplasia in a United States population. Cancer Causes Control 11: 713-720, 2000.

33. Mizobuchi S, Furihata M, Sonobe H, et al: Association between p53 immunostaining and cigarette smoking in squamous cell carcinoma of the esophagus. Jpn J Clin Oncol 30: 423-428, 2000 . 